\title{
3-D facial changes as a result of orthodontic treatment
}

The three-dimensional effects of orthodontic treatment on the facial soft tissues - a preliminary study.

\section{L. S. F. H. Ismail and J. P. Moss Br Dent J 2002; 192: 104-108}

\section{Objective}

To examine the three-dimensional effects of extraction and nonextraction orthodontic treatment on the facial soft tissues.

\section{Setting}

Department of Orthodontics, Royal London Hospital.

\section{Design}

Longitudinal study of two prospective cohorts of patients who were attending for orthodontic treatment.

\section{Method}

12 patients were included in each group, in accordance with the selection criteria. Three-dimensional optical surface scans of these patients were obtained in the one month prior to having fixed appliances placed and this was repeated at the end of treatment.

\section{Results}

The average face of the non-extraction patients was of greater general dimensions than the extraction average, both at the start and the end of treatment. During the fixed appliance treatment, the vermilion of the upper lip became more protrusive to a similar extent in both groups in relation to the reference plane. The lower lip vermilion and the philtrum showed no change for either group over the treatment time. The labiomental fold area showed a slightly greater forward movement in the extraction group with treatment.

\section{Conclusion}

Three-dimensional optical surface scanning allows data from the whole of the face to be assessed, as opposed to the lateral profile view used in the majority of the studies to date. The effects of the two types of treatment on the facial soft tissues were very similar, indicating that orthodontic treatment involving the extraction of teeth does not have a detrimental effect on the face.

\section{IN BRIEF}

Orthodontic treatment can affect the appearance of the face in all three dimensions of space. The extent of these effects can be accurately assessed using an imaging technique such as threedimensional optical surface scanning.

It is evident from this study that three-dimensional optical surface scanning allows a greater degree of assessment of facial changes occurring with orthodontic treatment than two-dimensional analyses. This non-invasive three-dimensional technique has great potential for further similar studies.

1 It is important (in particular in this aspect of orthodontics) that the profession make use of the best available resources to allow informed decisions on treatment to be reached.

\section{COMMENT}

Does orthodontic treatment adversely effect the face ie flatten the profile? More specifically, does orthodontic treatment including mid-arch extractions flatten the profile? Judging by the number of column inches in this and other journals, the effect of orthodontic treatment on the face is probably one of the most discussed subjects in the field of orthodontics at present.

The assessment of orthodontic treatment on the skeletal, dental and soft tissues has been, and still is, usually measured using lateral cephalograms. This produces data for the midsagittal plane and is effectively a 2-D analysis of a complex 3-D shape. The authors in this study have used the technique of Optical Surface Scanning (OSS) to analyse the changes in the facial soft tissues in 3-D for two groups of patients undergoing orthodontic treatment with or without extractions. OSS is one of a number of techniques for imaging the face in 3-D and one of the authors (JPM), apart from being involved with the development of the technique, has considerable experience with its application.

What does this study tell us? Firstly, the average face for the two groups, before treatment was different ie the nonextraction face was larger in the vertical and lateral dimension. Secondly, the difference in the average face post treatment, for both groups, was limited to the lips and labiomental fold, which were only $1-3 \mathrm{~mm}$ further forward in the non-extraction group. However this, as the authors acknowledge, is not a perfect study; the groups were small and not closely matched; the age range was large ie $10-18$ years; no control (untreated) group was included and no details of the orthodontic status were given i.e. why and which teeth were extracted. This is one of a small number of studies to look at the face in 3-D. It is clear that more research is needed but at least it is a step in the right direction.

Mr Robert D Evans

Consultant/Senior Lecturer in Orthodontics Great Ormond Street Hospital NHS Trust GKT Dental Institute 